\title{
Hydrophobic Segregation, Phase Transitions and the Anomalous Thermodynamics of Water/Methanol Mixtures
}

\author{
Tod A. Pascal ${ }^{*} \dagger$ and William A Goddard, III*, ${ }^{\dagger, \dagger}$ \\ ${ }^{\dagger}$ Materials and Process Simulation Center, California Institute of Technology, Pasadena, California 91125, United States \\ ${ }^{\star}$ World Class University Professor, Korea Advanced Institute of Science and Technology (KAIST), Daejeon, Korea
}

ABSTRACT: When water and methanol are mixed, the entropy of mixing decreases, whereas mixing simple liquids normally leads to an increase in entropy. One speculation on the origin of the anomaly involves formation of water icebergs next to the hydrophobic methanol group, while more recent theories point to nanoscale clustering of methanol molecules. To elucidate the origin of this effect, we carried out extensive molecular dynamics calculations on water/methanol mixtures ranging from 0 to $100 \%$ and applied the $2 \mathrm{PT}$ method to extract the entropy and free energy changes of each component as a function of concentration. We find that water molecules lose at most $1 / 35$ of their liquid
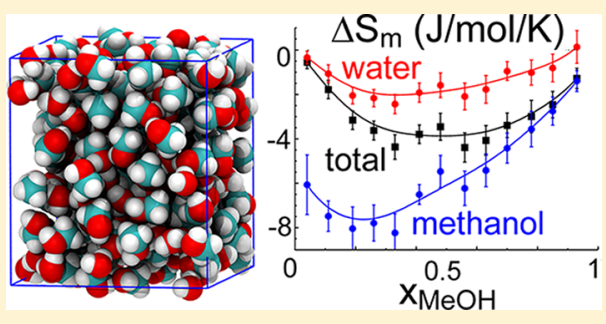
entropy in mixtures. Methanol molecules, on the other hand, lose 3 times as much entropy as the water molecules, and their rotational entropy contains the signature of the entropic loss. We find that methanol has a discontinuous specific heat profile in these mixtures with a maximum at $40 \%$ methanol. These results do not support the iceberg model of immobilized waters and instead suggests a molecular mechanism of hydrophobic segregation at low methanol concentration where ordering of the methanol molecules bury the hydrophobic group away from the water phase. For higher methanol concentrations, there is insufficient water to accomplish this effect, and the system freely mixes and transitions to one better described as water dissolved into methanol.

\section{INTRODUCTION}

The solubility and mixing of organics in water plays a critical role in biological life on this planet, and solvent extraction techniques underlie a plethora of industrial processes including many in the beer and wine industries. Mixtures of alcohols such as methanol and ethanol with water would appear to be the simplest examples of solvated systems with "hydrophobic" groups. However, these mixtures show unusual entropy and free energy with increasing alcohol concentration ${ }^{1}\left(x_{\mathrm{MeOH}}\right)$.

When two ideal liquids $i$ and $j$ are mixed, the entropy of mixing $S_{\text {mix }}$ necessarily increases, ${ }^{2}$ i.e.:

$$
S_{\text {mix }}=-k N\left(x_{i} \ln x_{i}+x_{j} \ln x_{j}\right)>-k N\left(x_{i}+x_{j}\right)
$$

since the mole fraction (number density) $x_{i}$ of each component is less than unity ( $N=\sum_{i} N_{i}$ is the total number of molecules and $k$ is Boltzmann's constant). However, for mixtures of methanol and water the entropy decreases, a fact that has been known experimentally as early as 1937 from the partial pressure measurements of Butler et al., ${ }^{3}$ who showed that excess free energy has a harmonic dependence on $x_{\mathrm{MeOH}}$. Further early experimental evidence came from the studies of Lama and $\mathrm{Lu},{ }^{4}$ who showed that the entropy of mixing dominates the thermodynamics while remaining always negative over the entire concentration range.

This anomalous thermodynamic behavior has stimulated numerous theoretical investigations, the earliest of which are the studies of Frank and Evans ${ }^{5}$ who introduced the so-called "Iceberg" model of solvation in 1945. This model posits that the negative entropy of water/methanol mixtures arise from the immobilization of the waters molecules solvating the hydrophobic $-\mathrm{CH}_{3}$ groups of methanol. Some recent experimental results ${ }^{6}$ have supported this model; however, it has been challenged both experimentally ${ }^{7-10}$ and theoretically (for example see refs 11 and 12). A more recent explanation suggests that incomplete mixing and molecular segregation is responsible for the entropy profile. ${ }^{12}$

Previous theoretical works have used molecular dynamics (MD) and Monte Carlo simulations to elucidate the hydrogen bonding, diffusion, and partial volumes effects of water/ methanol mixtures (see ref13 and the references therein). There have also been numerous studies of the enthalpy of mixing (see for example refs 14-17). Far less attention has been paid to the entropy and free energy of mixing, ${ }^{12,18,19}$ however. In this paper we report the excess entropy, enthalpy, and heat capacity of water/methanol mixtures at 17 points along the concentration curve, and we report separately the water and methanol contributions to these thermodynamics properties. Our studies use MD simulations and the two-phase thermodynamics $^{20,21}(2 \mathrm{PT})$ method.

\section{THEORY AND COMPUTATIONAL METHODS}

2.1. The 2PT Method Applied to Polymolecular Systems. Methods of efficiently accessing entropies of water from molecular dynamics trajectories ${ }^{22,23}$ are becoming more

Received: September 29, 2012

Revised: November 5, 2012

Published: November 5, 2012 
numerous and are providing intriguing insights into the physics that determine stability. One such method is the two-phase thermodynamics method of Lin, Blanco, and Goddard, ${ }^{20,21}$ which estimates the absolute entropies and quantum corrections (zero-point energy and heat capacity) to the enthalpy from short (typically 20 ps) MD trajectories. A key advantage of $2 \mathrm{PT}$ is that it relies solely on the atomic velocities, meaning that this can be applied straightforwardly to arbitrary systems, $^{24-26}$ systems under confinement, ${ }^{27,28}$ and dynamics over different time regimes. ${ }^{29-31}$

In $2 \mathrm{PT}$, the system thermodynamics are calculated from the density of states $\operatorname{DoS}(v)$ (spectral density or power spectrum), obtained from the Fourier transform of the velocity autocorrelation function $C(t):{ }^{32}$

$$
\begin{aligned}
& C(t)=\sum_{j=1}^{N} \sum_{k=1}^{3} m_{j}\left[\lim _{t \rightarrow \infty} \frac{1}{2 \tau} \int_{-\tau}^{\tau} v_{j}^{k}\left(t^{\prime}+t\right) v_{j}^{k}\left(t^{\prime}\right) \mathrm{d} t^{\prime}\right] \\
& \operatorname{DoS}(v)=\lim _{t \rightarrow \infty} \frac{1}{2 k T} \int_{-\tau}^{\tau} C(t) \mathrm{e}^{-2 \pi v t} \mathrm{~d} t
\end{aligned}
$$

The total $\operatorname{DoS}(v)$ is then partitioned into a contribution arising from pure diffusion in the liquid $\left[\operatorname{DoS}_{\text {diffuse }}(v)\right]$ and a contribution arising from solid-like vibrations $\left[\operatorname{DoS}_{\text {solid }}(v)\right]$, as proposed by Eyring and Ree: ${ }^{33}$

$$
\operatorname{DoS}(v)=f \times \operatorname{DoS}_{\text {diffuse }}(v)+(1-f) \times \operatorname{DoS}_{\text {solid }}(v)
$$

where $f$ is the "fluidicity factor": the fraction of the modes of the system that are diffusional. This $f$ factor is a function of the system properties (self-diffusion, density, and temperature) and is solved self-consistently from the MD trajectory. The total system thermodynamics is then recovered by integrating the individual power spectrum with the appropriate weighting factors, obtained from the Carnahan-Sterling (CS) equation of state (EOS) of hard-spheres ${ }^{34}$ in the case of $\operatorname{DoS}_{\text {diffuse }}(v)$ and from the Debye theory of a vibrating crystal ${ }^{2}$ in the case of $\operatorname{DoS}_{\text {solid }}(v)$.

The 2PT method was first validated by calculating the thermodynamics of Lennard-Jones systems, ${ }^{35}$ showing excellent agreement with Monte Carlo simulations. It has since been extended to molecular systems, predicting the thermodynamics of organic liquids, ${ }^{25}$ carbon dioxide over the entire phase diagram, ${ }^{24}$ of water from the vapor point to the critical point ${ }^{20}$ and in solvating hydrophobic and polar solutes ${ }^{36,37}$ with accuracy comparable to thermodynamic integration.

Application to heterogeneous systems is also straightforward. The $\operatorname{DoS}_{\text {solid }}(v)$ already treats the vibrations of each atom independently, so the only possible issue concerns the diffusional component. The original formalism determined the fluidicity by relating the measured system diffusivity $D(T, \rho)$ to the diffusivity of a gas of hard-spheres $D^{\mathrm{HS}}$ at the same temperature $(T)$ and density $(\rho)$, according to ChapmanEnskog theory: ${ }^{2}$

$$
D^{\mathrm{HS}}\left(T, f \rho, \sigma^{\mathrm{HS}}\right)=\frac{3}{8} \frac{1}{f \rho \sigma^{\mathrm{HS}}}\left(\frac{k T}{\pi m}\right)^{1 / 2} \frac{4 f y}{z(f y)-1}
$$

where $\sigma^{\mathrm{HS}}$ is the hard sphere diameter, $y=(\pi / 6) \rho \sigma^{\mathrm{HS}}{ }^{3}$ is the hard sphere packing fraction, and $z$ is the compressibility as defined by the Carnahan-Sterling EOS:

$$
z(y)=\frac{1+y+y^{2}-y^{3}}{(1-y)^{3}}
$$

For a heterogeneous system, one may consider that the diffusive component of each molecular species has a different hard sphere diameter $\sigma_{i}$ and thus packing fraction. In that case, the generalized Mansoori-Carnahan-Starling-Leland ${ }^{38}$ (MCSL) EOS for multicomponent hard sphere should be employed

$$
\begin{aligned}
& z(y)=\frac{1+y\left(1-3 \xi_{1}\right)+y^{2}\left(1-3 \xi_{2}\right)-y^{3} \xi_{3}}{(1-y)^{3}} \\
& y=\sum_{i=1} \frac{1}{6} \pi \rho \sigma_{i}^{3} x_{i}
\end{aligned}
$$

where $\rho$ is the number density, $x_{i}$ is the mole fraction of the $i$ th component, and $\xi_{i}$ are compressibility factors:

$$
\begin{aligned}
& \xi_{1}=\sum_{i=1} \sum_{j>i} \Delta_{i j}\left(\sigma_{i}+\sigma_{j}\right) / \sqrt{\sigma_{i} \sigma_{j}} \\
& \xi_{2}=\sum_{i=1} \sum_{j>i} \Delta_{i j} \sum_{k=1}\left(\frac{y_{k}}{y}\right) \frac{\sqrt{\sigma_{i} \sigma_{j}}}{\sigma_{k}} \\
& \xi_{3}=\left[\sum_{i=1}\left(\frac{y_{i}}{y}\right)^{2 / 3} x_{i}^{1 / 3}\right]^{3}
\end{aligned}
$$

where

$$
\Delta_{i j}=\frac{\sqrt{y_{i} y_{j}}}{y} \frac{\left(\sigma_{i}-\sigma_{j}\right)^{2}}{\sigma_{i} \sigma_{j}} \sqrt{x_{i} x_{j}}
$$

In practice, the excess properties of the MCSL EOS over the original CS EOS are found to be small, ${ }^{39}$ and assumptions of ideal mixing lead to accurate predictions of the properties of the mixture, ${ }^{40}$ i.e.

$$
z(y)=\sum_{i=1} x_{i} z_{i}(y)
$$

where $z_{i}(y)$ is the compressibility factor each species defined in eq 6, assuming the same hard sphere diameter. Thus, the $2 \mathrm{PT}$ formalism is extendable to arbitrary mixtures of polymolecular systems by simply weighting the compressibility factors by the mole fraction. ${ }^{41}$

2.2. MD Simulations Procedure. Pre-equilibrated boxes of methanol (described with the all-atom OPLS AA/ $\mathrm{L}^{42,43}$ forcefield), and SPC/E, ${ }^{44} \mathrm{SPC} / \mathrm{Fw}^{45}{ }^{4} \mathrm{TIP} 4 \mathrm{P},{ }^{46}$ and TIP4$2005^{47}$ water molecules were simulated for $2.5 \mathrm{~ns}$ of constant pressure (1 atm), constant temperature $(298 \mathrm{~K})$, and (NPT) dynamics using the LAMMPS ${ }^{48}$ simulation engine. The OPLS AA/L forcefield for methanol was selected as it has been fitted to reproduce the liquid free energy and various thermodynamic and structural properties at room temperature. We used a Nose-Hoover thermostat with a temperature coupling constant of 0.1 ps and a barostat with a pressure piston constant was 2.0 ps. The integration time step was set to $1 \mathrm{fs}$, and bonds involving hydrogens and the $\mathrm{HOH}$ angle on the water molecules were constrained by the SHAKE ${ }^{49}$ algorithm, except in the case of the SPC/Fw water model, where the bonds and angles on the waters were kept flexible. The equations of motion used are those of Shinoda et al., ${ }^{50}$ which combine the hydrostatic equations of Martyna et al. ${ }^{51}$ with the strain energy proposed by Parrinello and Rahman. ${ }^{52}$ The time integration schemes closely follow the time-reversible measure-preserving 
Table 1. Structural Properties and Absolute Thermodynamics of the Pure Liquids Used in This Study

\begin{tabular}{|c|c|c|c|c|c|c|c|}
\hline \multirow[b]{2}{*}{ property } & \multicolumn{2}{|c|}{ methanol } & \multicolumn{5}{|c|}{ water } \\
\hline & $\exp$ & OPLS/aa & $\exp$ & $\mathrm{SPC} / \mathrm{E}$ & $\mathrm{SPC} / \mathrm{Fw}$ & TIP4P & TIP4-2005 \\
\hline$m_{\mathrm{v}}(\mathrm{cc})^{a}$ & 67.3 & $69.6 \pm 1.2$ & 30.0 & $30.0 \pm 0.4$ & $29.8 \pm 0.5$ & $30.7 \pm 0.5$ & $30.2 \pm 0.4$ \\
\hline$D\left(\times 10^{-5} \mathrm{~cm}^{2} / \mathrm{s}\right)^{b}$ & 2.2 & $3.1 \pm 0.5$ & 2.3 & $2.6 \pm 0.2$ & $2.4 \pm 0.2$ & $3.9 \pm 0.4$ & $2.1 \pm 0.2$ \\
\hline$H^{0}(\mathrm{~kJ} / \mathrm{mol})^{c}$ & 9.2 & $11.6 \pm 0.3$ & -34.1 & $-36.9 \pm 0.1$ & $-30.5 \pm 0.3$ & $-30.9 \pm 0.1$ & $-37.1 \pm 0.1$ \\
\hline$S^{0}(\mathrm{~J} / \mathrm{mol} / \mathrm{K})^{d}$ & 127.2 & $120.8 \pm 1.3$ & 69.9 & $60.1 \pm 0.5$ & $61.7 \pm 0.6$ & $64.5 \pm 0.6$ & $57.3 \pm 0.5$ \\
\hline$G^{0}(\mathrm{~kJ} / \mathrm{mol})^{e}$ & -28.7 & $-24.4 \pm 0.6$ & -55.9 & $-49.7 \pm 0.1$ & $-48.5 \pm 0.5$ & $-50.0 \pm 0.1$ & $-54.4 \pm 0.2$ \\
\hline$C_{\mathrm{v}}(\mathrm{J} / \mathrm{mol} / \mathrm{K})^{f}$ & 67.6 & $56.0 \pm 0.3$ & 74.5 & $76.0 \pm 1.0$ & $66.7 \pm 1.2$ & $68.5 \pm 1.2$ & $76.3 \pm 1.1$ \\
\hline
\end{tabular}

${ }^{a}$ Molar volume. Experimental densities $\rho\left(\mathrm{g} / \mathrm{cm}^{3}\right)$ from ref $67 ; m_{\mathrm{v}}(\mathrm{cc})=m_{\mathrm{m}}(\mathrm{g}) /(\rho \times 0.6023), m_{\mathrm{m}}$ is the molar mass. ${ }^{b}$ Self diffusion constant. Experimental values from ref $68 .{ }^{c}$ Enthalpy. Experimental values from refs 67 and 69 which gives $\Delta H_{\text {liq }}$ which converts to $H^{0}$ by the isothermal work required to bring the gas to liquid density $H^{0}=\Delta H_{\mathrm{liq}}+(P V=R T=2.48 \mathrm{~kJ} / \mathrm{mol}) .{ }^{d}$ Standard molar entropy. Experimental values from refs 67 and 69. ${ }^{e}$ Gibbs free energy $=\mathrm{H}^{0}-T S^{0} .{ }^{f}$ Constant volume heat capacity. Experimental values from refs 67 and 69 . Reference 67 gives $C_{\mathrm{p}}: C_{\mathrm{v}}=C_{\mathrm{p}}-$ $V T\left(\alpha_{\mathrm{p}}^{2} / \kappa_{\mathrm{T}}\right)$, where $\alpha_{\mathrm{p}}$ is the coefficient of thermal expansion and $\kappa_{\mathrm{T}}$ is the isothermal compressibility, both calculated from NPT dynamics.
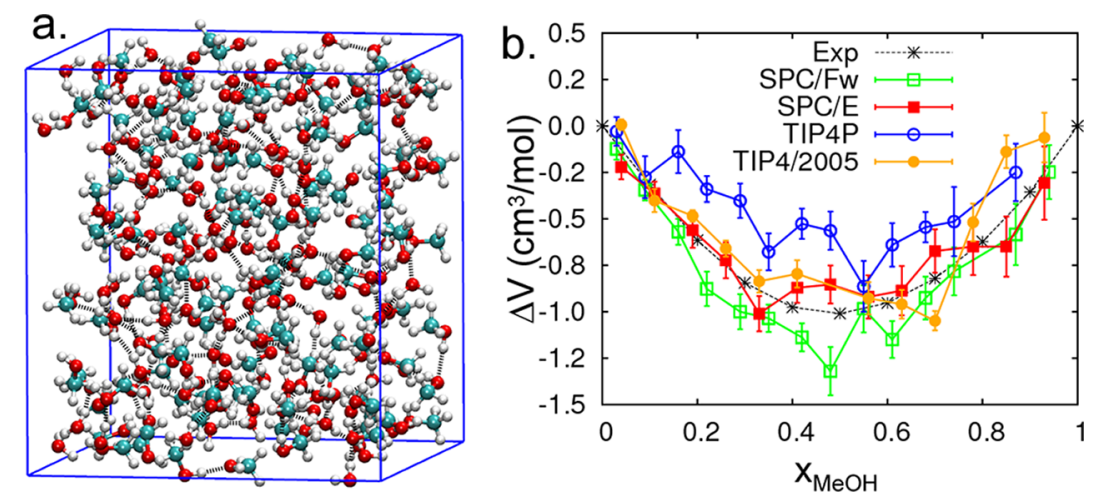

Figure 1. (a) Final snapshot of water/methanol system at $x_{\mathrm{MeOH}}=0.56$ after $2 \mathrm{~ns}$ of NPT dynamics at $298 \mathrm{~K}$ and 1 atm using the all-atom OPLS methanol forcefield and the SPC/E water model. The unit cell measures $2.03 \times 2.31 \times 2.32 \mathrm{~nm}^{3}$. Hydrogen bonding is indicated by dashed black lines. (b) Excess molar volumes of water/methanol mixtures at $298 \mathrm{~K}$ and $1 \mathrm{~atm}$. Simulations using the SPC/Fw (green open squares), SPC/E (red filled squares), TIP4P (blue open circles), and TIP4-2005 (orange filled circles) are compared to the experiment (stars over black dashed line). Each calculated value is the average of 15 independent 20 ps trajectories taken at 100 ps intervals from the last $1.5 \mathrm{~ns}$ of a $2.5 \mathrm{~ns}$ NPT simulation. Uncertainties in our calculations are indicated by vertical errorbars.

Verlet integrators derived by Tuckerman et al. ${ }^{53}$ Long-range Coulombic interactions were calculated using the particleparticle particle-mesh Ewald method ${ }^{54,55}$ (with a precision of $10^{-5} \mathrm{kcal} / \mathrm{mol}$ ), while the van der Waals interactions were computed with a cubic spline (inner cutoff of $9 \AA$ and an outer cutoff of $10 \AA$ ).

Mixtures were generated by randomly replacing required numbers of methanol molecules with water to achieve a total of 216 molecules. Each mixture was then subjected to a further 2.5 ns MD simulation. In total, 17 simulations of 2.5 ns were run for each of the four water models, for a total of $96 \mathrm{CPU}$ hours on a dual core Intel Xenon $2.7 \mathrm{GHz}$ processor. Due to the efficiency LAMMPS and the 2PT method, we will show that $2.5 \mathrm{~ns}$ is sufficient to obtain converged thermodynamics in excellent agreement with experiments.

2.3. Thermodynamics Calculations. Snapshots of the system were saved every 100 ps for post trajectory analysis with the 2PT method. Each snapshot was then subjected to an additional 20 ps of constant volume, constant energy (NVE) dynamics, saving the atomic energy, coordinates and velocities every 4 fs. The thermodynamic variables were averaged over the last 15 snapshots ( $1.5 \mathrm{~ns})$, and the statistical variance was recorded. This represents $11 \mathrm{~h}$ of additional CPU time for the 68 systems considered in this work, or an $11 \%$ increase. This additional cost arises because we chose not to save the original trajectory at steps of 4 fs required for the $2 \mathrm{PT}$ analysis which required tremendous additional disk storage. Except for this, there would be negligible additional cost. An alternative would be to do the $2 \mathrm{PT}$ calculation on the fly, which we chose not to do. This means that our approach is orders of magnitude more efficient than those based on thermodynamics integration ${ }^{56,57}$ or Widom particle insertion. ${ }^{58}$

\section{RESULTS AND DISCUSSION}

3.1. Performance of Different Water Models and Comparison to Experiment. The excess properties $P_{\mathrm{ex}}$ are determined as the difference between the water and methanol molecules in the mixture $P^{\text {mix }}$ and the average value of each molecule in the pure liquid $\widehat{P^{\text {pure }}}$ :

$$
\begin{aligned}
P_{\mathrm{ex}}= & x_{\mathrm{MeOH}}\left(\frac{P_{\mathrm{MeOH}}^{\mathrm{mix}}}{N_{\mathrm{MeOH}}}-\widehat{P_{\mathrm{MeOH}}^{\mathrm{pure}}}\right) \\
& +\left[1-x_{\mathrm{MeOH}}\right]\left(\frac{P_{\mathrm{WAT}}^{\mathrm{mix}}}{N_{\mathrm{WAT}}}-\widehat{P_{\mathrm{WAT}}^{\text {pure }}}\right)
\end{aligned}
$$

Thus, we first tested the performance of our simulation methodology and the $2 \mathrm{PT}$ method in predicting the properties of the pure liquids. As shown in Table 1, the agreement to experiment is very good to excellent. The molar volumes are calculated to within $3 \%$ for methanol and $0.5 \%$ for water over all water models, consistent with the parametrization of the forcefields and other published results. ${ }^{59}$ There is, however, a slight $(<5 \%)$ overestimation of the diffusion constants in this 

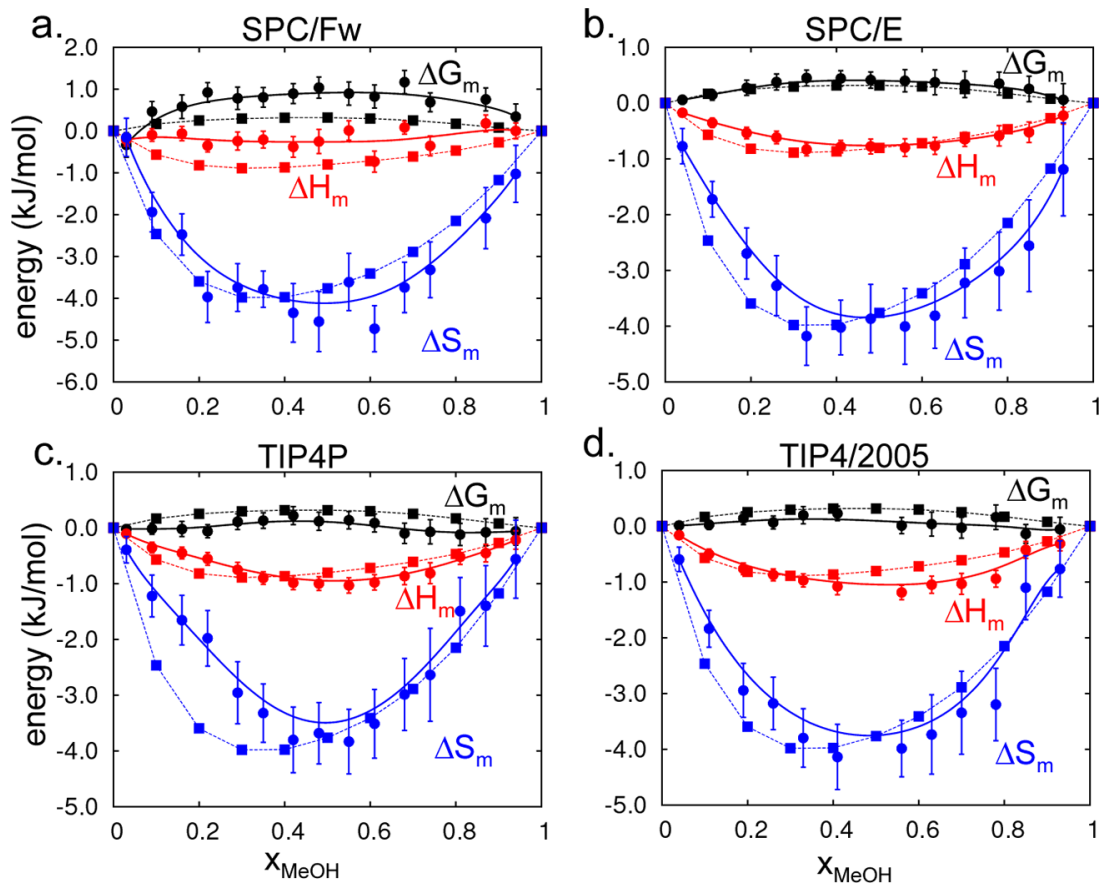

Figure 2. Excess thermodynamics of water/methanol mixtures at $298 \mathrm{~K}$ and $1 \mathrm{~atm}$. The calculated (solid lines with circles) and experimental (dashed lines with squares) molar enthalpy ( $\mathrm{red}-\mathrm{kJ} / \mathrm{mol}$ ), entropy (blue $-\mathrm{J} / \mathrm{mol} / \mathrm{K}$ ) and Gibbs free energy (black - $\mathrm{kJ} / \mathrm{mol}$ ) are presented for the (a) SPC/Fw, (b) SPC/E, (c) TIP4P, and (d) TIP4-2005 water models. The data points are fit to a bezier function (lines) for presentation purposes. Overall we find good agreement with experiments over the entire concentration range, with the SPC/E water model giving the best agreement.

work compared to that in published results, which arises from the difference in assessing mean-squared displacements from long ( $>100 \mathrm{ps})$ trajectories and the method used in this study which relies on short (20 ps trajectories) and the Green-Kubo method. ${ }^{25}$ The thermodynamic properties are all reasonably reproduced, although the standard molar entropy is slightly underestimated compared to that in experiments. Part of this arises from the general tendency of the 2PT method to underestimate entropies by $2 \%$ compared to thermodynamic integration, ${ }^{30}$ although most of the disagreement with experiment reflects inadequacies in the parametrization of the forcefield. $^{20}$

In Figure $1 \mathrm{~b}$ we calculate the excess volume of the water/ methanol mixtures with the various water forcefields. We find good agreement with experiments over the entire concentration range. The TIP4-2005 and SPC-E water models show the best agreement, with average errors of $-3.4 \%$ and $-1.5 \%$, respectively. The SPC-Fw water model generally overestimates the excess volume $(+8.3 \%)$, while the TIP4P water model generally underestimates it $(-12.7 \%)$. Similar to our results, Gonzalez-Salgado and Nezbeda ${ }^{13}$ performed MD simulations using the united-atom OPLS methanol forcefield and the TIP4P water model and found that the excess volumes agree well with experiments. Our results are also in general agreement with the work of Wensink, ${ }^{17}$ who used the all-atom OPLS methanol forcefield and the TIP4P water model.

The excess thermodynamics also show good agreement with experiments, although here we find more pronounced differences between that various water models. For example, the calculated excess enthalpy of the methanol/SPC-Fw system is underestimated (more positive) by approximately 50\% compared to experiment (Figure 2a). We find slightly overestimated (more negative) excess entropy losses compared to experiment, and a predicted minimum $x_{\mathrm{MeOH}}=0.55$ that is shifted to the right of the experiment $x_{\mathrm{MeOH}}=0.32$. Thus, while the excess free energy shows the correct behavior, it is overestimated by $35 \%$.

The rigid SPC-E water model (Figure $2 \mathrm{~b}$ ) performs better that the flexible SPC-Fw, with excellent agreement found in the excess enthalpy (average overestimated of $1.7 \%$ ), entropy $(+2.1 \%)$, and free energy $(+0.7 \%)$. Similar to SPC-Fw however, the minimum in the excess entropy distribution $x_{\mathrm{MeOH}}=0.49$ is shifted to the right of experiments. We note that the SPC-Fw was parametrized to have the same bulk behavior as the SPC-E water model (Table 1); hence, its significantly worse performance in methanol mixtures is somewhat surprising. This possibly indicates that the thermodynamics are rather sensitive to the constraints employed by the SHAKE algorithm. Thus, care should be taken when interpreting energies for heterogeneous system with constrained hydrogens on one set of molecules (methanol) and not the other (water).

In general, the rigid TIP water models (Figure $2 \mathrm{c}, \mathrm{d}$ ) show worse agreement with experiment than the SPC-E water model. We find that the excess enthalpy is underestimated at low concentrations, matches the experiment at $x_{\mathrm{MeOH}} \approx 0.4$, and is overestimated thereafter. The minimum in the excess enthalpy curve is again shifted to the right of experiment $x_{\mathrm{MeOH}}=0.42$, occurring at $x_{\mathrm{MeOH}}=0.62$ and 0.66 for TIP4P and TIP4-2005, respectively. The excess entropic loss is underestimated by $-10.2 \%$ and $-4.8 \%$, respectively, with the minimum occurring at $x_{\mathrm{MeOH}} \approx 0.53$. Thus, the excess free energy is overestimated by $+23 \%$ and $+16 \%$, respectively.

The right shifting of the minimum in the excess entropy curve compared to experiments suggests deficiencies in the forcefields just in this study. Since both the water and methanol forcefields do a reasonable job of describing the entropy of the pure liquids, this rightward shift may be attributed to the incorrect methanol/water nonbond interactions, which were 
Table 2. Excess Thermodynamics of Water/Methanol Mixtures over the Entire Concentration Rang Calculated in This Study Compared to Experiment, ${ }^{3,4,70}$ Monte Carlo Simulation of Tanaka and Gubbins ${ }^{18}(\mathrm{TG})^{a}$

\begin{tabular}{|c|c|c|c|c|c|c|c|}
\hline$x_{\mathrm{MeOH}}$ & energy $^{b}$ & expt & TIP $4 \mathrm{P}^{c}$ & TIP4-2005 ${ }^{c}$ & $\mathrm{SPC} / \mathrm{E}^{c}$ & $\mathrm{SPC} / \mathrm{Fw}^{c}$ & T.G. ${ }^{d}$ \\
\hline \multirow[t]{3}{*}{0.05} & $\Delta H_{\mathrm{m}}\left(\Delta E_{\mathrm{tot}}\right)$ & -0.57 & $\begin{array}{r}-0.09 \pm 0.05 \\
(-0.00 \pm 0.7)\end{array}$ & $\begin{array}{c}-0.20 \pm 0.04 \\
(-0.12 \pm 0.05)\end{array}$ & $\begin{array}{c}-0.18 \pm 0.07 \\
(-0.06 \pm 0.04)\end{array}$ & $\begin{array}{c}-0.22 \pm 0.14 \\
(-0.09 \pm 0.09)\end{array}$ & $(-0.25)$ \\
\hline & $\Delta S_{\mathrm{m}}$ & -2.48 & $-0.33 \pm 0.24$ & $-0.81 \pm 0.17$ & $-0.70 \pm 0.31$ & $-0.36 \pm 0.50$ & -1.01 \\
\hline & $\Delta G_{\mathrm{m}}$ & 0.17 & $-0.00 \pm 0.08$ & $0.02 \pm 0.07$ & $0.06 \pm 0.06$ & $-0.24 \pm 0.32$ & 0.05 \\
\hline \multirow[t]{3}{*}{0.27} & $\Delta H_{\mathrm{m}}\left(\Delta E_{\mathrm{tot}}\right)$ & -0.88 & $\begin{array}{c}-0.77 \pm 0.13 \\
(-0.58 \pm 0.14)\end{array}$ & $\begin{array}{c}-0.87 \pm 0.10 \\
(-0.77 \pm 0.11)\end{array}$ & $\begin{array}{c}-0.66 \pm 0.11 \\
(-0.20 \pm 0.08)\end{array}$ & $\begin{array}{c}-0.33 \pm 0.20 \\
(-0.93 \pm 0.12)\end{array}$ & $(-0.63)$ \\
\hline & $\Delta S_{\mathrm{m}}$ & -3.93 & $-3.07 \pm 0.48$ & $-3.11 \pm 0.44$ & $-3.31 \pm 0.55$ & $-3.95 \pm 0.55$ & -3.42 \\
\hline & $\Delta G_{\mathrm{m}}$ & 0.29 & $0.17 \pm 0.13$ & $0.03 \pm 0.10$ & $0.37 \pm 0.15$ & $0.80 \pm 0.26$ & 0.70 \\
\hline \multirow[t]{3}{*}{0.54} & $\Delta H_{\mathrm{m}}\left(\Delta E_{\mathrm{tot}}\right)$ & -0.79 & $\begin{array}{c}-0.98 \pm 0.12 \\
(-0.84 \pm 0.11)\end{array}$ & $\begin{array}{c}-1.18 \pm 0.13 \\
(-1.08 \pm 0.15)\end{array}$ & $\begin{array}{c}-0.82 \pm 0.18 \\
(-0.26 \pm 0.08)\end{array}$ & $\begin{array}{c}-0.35 \pm 0.30 \\
(-1.12 \pm 0.17)\end{array}$ & $(-0.48)$ \\
\hline & $\Delta S_{\mathrm{m}}$ & -3.69 & $-3.49 \pm 0.58$ & $-3.86 \pm 0.53$ & $-3.93 \pm 0.75$ & $-4.60 \pm 0.62$ & -3.50 \\
\hline & $\Delta G_{\mathrm{m}}$ & 0.31 & $0.05 \pm 0.12$ & $-0.02 \pm 0.20$ & $0.42 \pm 0.20$ & $1.03 \pm 0.24$ & 0.72 \\
\hline \multirow[t]{3}{*}{0.70} & $\Delta H_{\mathrm{m}}\left(\Delta E_{\mathrm{tot}}\right)$ & -0.61 & $\begin{array}{c}-0.94 \pm 0.14 \\
(-0.78 \pm 0.15)\end{array}$ & $\begin{array}{c}-1.06 \pm 0.17 \\
(-0.92 \pm 0.15)\end{array}$ & $\begin{array}{c}-0.73 \pm 0.12 \\
(-0.20 \pm 0.07)\end{array}$ & $\begin{array}{c}0.04 \pm 0.16 \\
(-0.93 \pm 0.13)\end{array}$ & $(-0.39)$ \\
\hline & $\Delta S_{\mathrm{m}}$ & -2.89 & $-2.75 \pm 0.63$ & $-3.38 \pm 0.74$ & $-3.39 \pm 0.55$ & $-3.60 \pm 0.62$ & -2.77 \\
\hline & $\Delta G_{\mathrm{m}}$ & 0.25 & $-0.13 \pm 0.20$ & $-0.02 \pm 0.20$ & $0.35 \pm 0.19$ & $1.18 \pm 0.27$ & 0.61 \\
\hline
\end{tabular}

${ }^{a}$ Values in parentheses indicates MD energies. ${ }^{b}$ Excess molar enthalpy $\Delta H_{\mathrm{m}}(\mathrm{kJ} / \mathrm{mol})$. The $2 \mathrm{PT}$ method produces the internal energy $E$, which is related to the enthalpy by the work term $P V: H=E+(P V=R T=2.48 \mathrm{~kJ} / \mathrm{mol})$. We apply zero-point energy and thermal corrections to the $\mathrm{MD}$ total energy $\left(E_{\mathrm{md}}\right)$, so that $\Delta H=\Delta E_{\mathrm{md}}+\Delta \mathrm{ZPE}+T \Delta C_{\mathrm{v}}$. Excess molar entropy $\Delta S_{\mathrm{m}}(\mathrm{J} / \mathrm{mol} / \mathrm{K})$ and excess molar Gibbs free energy $\Delta G_{\mathrm{m}}(\mathrm{kJ} / \mathrm{mol})=$ $\Delta H_{\mathrm{m}}-T \Delta S_{\mathrm{m}} \cdot{ }^{c}$ This work: OPLS AA/L methanol model and the ${ }^{\mathrm{b}} \mathrm{TIP} 4 \mathrm{P}-2005$ and ${ }^{\mathrm{c}} \mathrm{SPC} / \mathrm{E}$ water models. ${ }^{d}$ Tanaka and Gubbins used the united atom OPLS methanol forcefield and the TIP4P water model. ${ }^{18}$
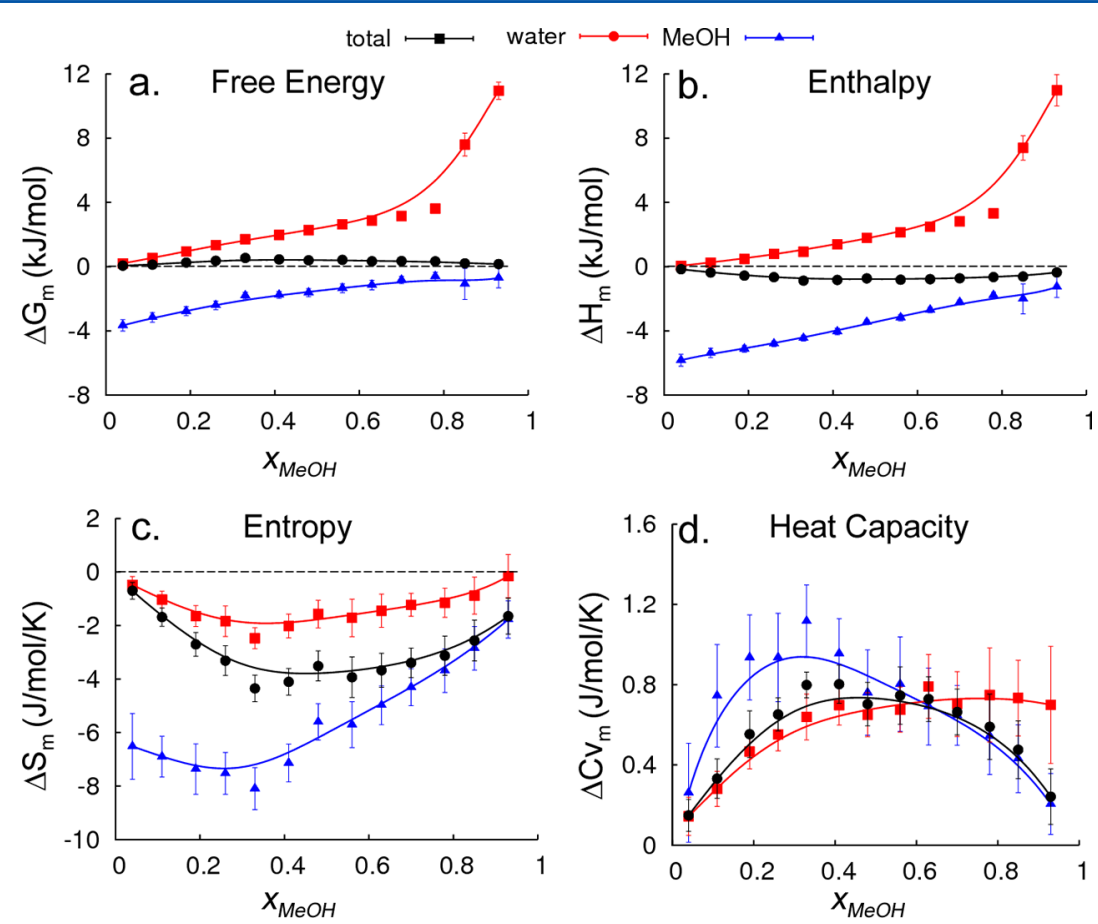

Figure 3. Components of excess thermodynamic quantities of the OPLS/SPC-E system with increasing methanol concentration. The total thermodynamics (black squares) is partitioned into contributions resulting from the water (red circles) and methanol (blue triangles) molecules. The excess molar (a) Gibbs free energy $\Delta G_{\mathrm{m}}(\mathrm{kJ} / \mathrm{mol})$. (b) Enthalpy $\Delta H_{\mathrm{m}}(\mathrm{kJ} / \mathrm{mol})$. (c) Entropy $\Delta S_{\mathrm{m}}(\mathrm{J} / \mathrm{mol} / \mathrm{K})$. (d) Constant volume heat capacity $\Delta C_{\mathrm{vm}}(\mathrm{J} / \mathrm{mol} / \mathrm{K})$ are presented. The solid lines are cubic spline fits for presentation purposes.

obtained from combination rules. This suggests a design principle for a more accurate forcefield by explicitly tuning the water/methanol nonbond interactions to better match the experimental entropy loss curve. Additional improvements could include charge polarization effects, ${ }^{60-62}$ which are ignored by our fixed charge forcefields.

3.2. Comparison to Other Published Computational

Results. Several authors have calculated the excess enthalpy of methanol/water mixtures using the OPLS methanol forcefield and the TIP4P water model. We highlight two such studies in order to place our results in proper context. Dai et al. ${ }^{14}$ used the united atom OPLS methanol forcefield with the TIP4P water model and calculated excess enthalpies of mixing along the concentration curve. We find very good agreement with their published results: -0.54 vs -0.66 for $x_{\mathrm{MeOH}}=0.2 ;-0.99$ vs -0.87 for $x_{\mathrm{MeOH}}=0.4 ;-1.00$ vs -0.63 for $x_{\mathrm{MeOH}}=0.6$; and 
$-0.56 \mathrm{vs}-0.33 \mathrm{~kJ} / \mathrm{mol}$ for $x_{\mathrm{MeOH}}=0.8$, respectively, although their excess enthalpies tend to be underestimated compared to ours and those of experiments. Interestingly, they too observe that the TIP4P water model tends to shift the minimum in the excess enthalpy curve to the right, further validating our simulation protocol.

When calculating our internal energies (enthalpies), we correct the $\mathrm{MD}$ energy for zero-point point energy (ZPE) motions and thermal (heat capacity) effects:

$$
E_{\text {tot }}=E_{\mathrm{md}}+E_{\mathrm{ZPE}}+T C_{\mathrm{v}}
$$

We propose that this corrected energy is more directly comparable to experiments as ZPE and thermal effects are inherent in any experimental measurement. Indeed, we find that the agreement with experiments improves significantly by using the corrected enthalpies than simply using the MD energy (Table 2) and that the results of Dai et al., who did not correct their energies, are in better agreement with our MD energies, thus accounting for the observed differences.

Tanaka and Gubbins ${ }^{18}$ used the united atom OPLS methanol forcefield and the TIP4P water model in Monte Carlo simulations and obtained enthalpies of mixing in good agreement with our results at low methanol concentrations, but their results are underestimated compared to ours and those of experiments at higher concentrations (Table 2). This discrepancy might arise from differences in the OPLS united atom and all atom forcefields, as well as in the methodology employed (Monte Carlo vs molecular dynamics, treatment of long-range electrostatics, cutoffs for van der Waals interactions, etc.). In spite of these differences, the overall agreement is quite reasonable. They also determined the excess free energy of mixing according to the Monte Carlo recursion method of $\mathrm{Li}$ and Scheraga. ${ }^{63}$ As shown in Table 2, we find very good agreement with their results over the entire concentration range, particularly at lower methanol concentrations where the water molecules dominate the thermodynamics.

3.3. Excess Entropy/Enthalpy Contributions of Water and Methanol. We will now restrict our discussion to the SPC/E water model as it gives the best agreement with experiment. Greater insight into the nature of the free energy change with increasing methanol concentration is obtained by separately considering the excess free energies of the methanol and water molecules, relative to those of the pure liquids. Such analysis is trivially accomplished using the $2 \mathrm{PT}$ method, and are shown in Figure 3. Thus, we find that the excess free energy of transferring methanol from the pure liquid into the mixture is negative (favorable), while that of water is positive (unfavorable). These opposing excess free energy profiles are compensatory and cancel almost exactly; however, the excess free energy loss of the waters is always slightly greater than the gain of the methanols, leading to a net unfavorable (positive) excess free energy of mixing profile. The excess free energy of both components decreases (becomes more positive) with increasing methanol concentration.

Analysis of the enthalpy reveals a story similar to that of the free energy: excess enthalpic gains of the stabilized methanol molecules in the mixture are reduced with increasing methanol concentration as is the excess enthalpy of the destabilized water. However, and unlike the excess free energy, the excess enthalpic gains of the methanol dominates the excess enthalpic loss of the waters, leading to a slight net increase (negative) excess enthalpy along the entire concentration curve. This means that the excess entropy is the dominant term in the excess free energy of this system.

The excess entropy of the water and methanol molecules with increasing methanol concentration is shown in Figure 2c, from which we make several general observations:

(1) Unlike the excess enthalpy profile, both methanol and water lose entropy in the mixtures compared to the pure liquids and have a parabolic profile with increasing methanol concentration.

(2) Water molecules lose at most $2 \mathrm{eu}(\mathrm{eu}=\mathrm{J} / \mathrm{mol} / \mathrm{K})$ of entropy at $x_{\mathrm{MeOH}}=0.35$, or $1 / 35$ of their total.

(3) Compared to the entropy loss of each water molecule, the entropy loss in each methanol molecule is considerably greater, with a maximum of 8 eu of entropy at $x_{\mathrm{MeOH}}=0.35$ or $1 / 15$ of the total.

The relatively modest reduction in the excess entropy of the water molecules with the mixtures (at most $1 / 35$ of the total in the pure liquid) does not support the idea of water "icebergs" around the hydrophobic groups of methanol. Rather, these results suggests the formation of "mini micelles", at least at low methanol concentrations. Soper et al. ${ }^{12}$ showed that the excess entropy results of Tanaka and Gubbins can be exactly recovered by an empirical model of the excess entropy of mixing (Table 2) where the only adjustable parameter is the width of the interfacial layer between clusters. This led to the suggestion that the observed negative excess entropy of water/ methanol mixtures arises naturally from the tendency of the molecules to segregate and form clusters. ${ }^{9,12,64}$ Our results are tacitly supportive of this interpretation, if not providing explicit evidence of it.

3.4. The Excess Heat Capacity of Mixing. The excess specific heat capacity of the water and methanol molecules in the mixtures is shown in Figure $3 \mathrm{~d}$. Consistent with the slightly reduced excess entropy, we find that the specific heat of each water molecule increases monotonically until $x_{\mathrm{MeOH}}=0.35$ and is fairly constant afterward. Thus, although the excess molar entropy of each water molecule is almost zero at high methanol concentrations, these waters appear to have a different hydrogen-bonding environment than in the pure liquid.

On the other hand, the excess specific heat capacity of the methanol molecules initially increases from zero, achieves a maximum at $x_{\mathrm{MeOH}}=0.4$, and then decreases monotonically to zero at higher methanol concentrations. We propose that this point represents a transition from water-rich to methanol-rich environment, a reasonable proposition considering that the molar volume of methanol is twice that of water. Of course, the experimental crossover point should occur at a somewhat lower methanol mole fraction than calculated here due to the shift in minimum excess entropy concentration as noted previously. Previous experimental neutron diffraction ${ }^{65}$ and computational studies have suggested an experimental crossover point of 0.23 $<x_{\mathrm{MeOH}}<0.54$.

3.5. Components of Excess Entropy Loss. What is the nature of the entropic loss in these water/methanol mixtures? To investigate this further, we decompose the total excess entropy $\Delta S^{\text {tot }}$ into contributions arising from the different types of molecular motions:

$$
\begin{aligned}
\Delta S_{\mathrm{m}}^{\text {tot }}= & \Delta S_{\mathrm{m}}^{\text {trans }}=\left(f \Delta S_{\mathrm{m}}^{\text {diffuse }}+[1-f] \Delta S_{\mathrm{m}}^{\text {trans }- \text { lib }}\right) \\
& +\Delta S_{\mathrm{m}}^{\text {rot }}+\Delta S_{\mathrm{m}}^{\text {ivib }}
\end{aligned}
$$




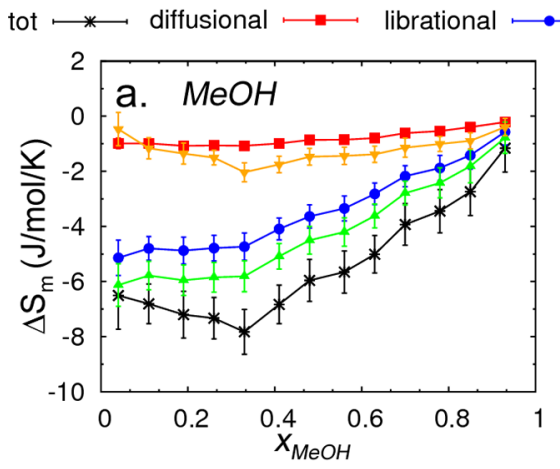

translational $\Longleftrightarrow$ rotational

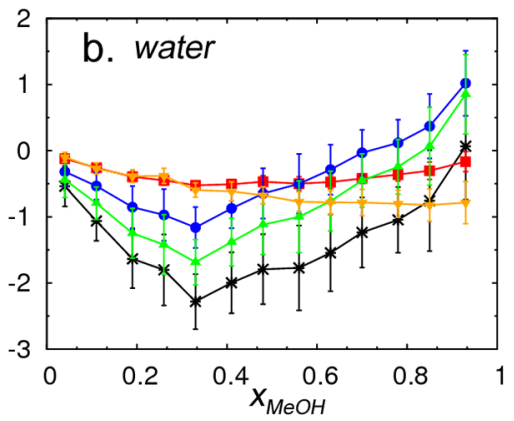

Figure 4. Components of the excess molar entropy with increasing methanol concentration. The total excess entropy $S_{\text {tot }}$ (black stars) is separated in translational $S_{\text {trans }}$ (green up triangles) and rotational $S_{\text {rot }}$ (orange down triangles) contributions: $S_{\text {tot }}=S_{\text {trans }}+S_{\text {rot }}+S_{\text {ivib. Note that the the internal }}$ vibrational component of the entropy $\Delta S_{\text {ivib }}$ is small and relatively constant in the case of $\mathrm{MeOH}$ and is not shown. Likewise, $\Delta S_{\text {ivib }}$ is zero for the rigid water molecules (due to SHAKE constraints), and very small for the SPC/Fw flexible water molecule. We further partition the translational entropy in diffusional $S_{\text {diffuse }}$ (red squares) and $S_{\text {lib }}$ librational (oscillatory motions as in a solid) (blue circles) contributions: $S_{\text {trans }}=S_{\text {diffuse }}+S_{\text {lib }}$. We separately analyze the (a) methanol and (b) water molecules.

where $f$ is the fluidicity factor in eq $4, S^{\text {trans }}$ is entropy arising from translations, defined as the sum of diffusional (center of mass motion) and librational (solidlike translation) entropies, $S^{\text {rot }}$ arise from rotations (angular velocity about the center of mass), and $S^{\text {ivib }}$ arises from internal molecules vibrations (bonds stretching, angle bending, torsional rotations). This decomposition is valid under the assumption of independent degrees of freedom.

As shown in Figure 4a, losses in the excess librational entropy accounts for $80 \%$ of the total excess entropy loss of methanol at low concentrations. We find that losses due to excess diffusional entropy are much less important $(<5 \%)$, suggesting that the methanol molecule loses entropy primarily by suppression of the low-frequency oscillatory (or rattling) motions present in the pure liquid, and not because of reduced diffusion. Consistent with the view of Soper et al., the flat profile in the librational entropy until $x_{\mathrm{MeOH}}=0.35$ can be interpreted as the formation of successively larger segregated structures of the same thermodynamic character. The decreased (less negative) excess entropic loss after $x_{\mathrm{MeOH}}=0.35$ would then represent a molecular crossover point from water-rich to methanol-rich mixtures suggested by the heat capacity. We find that losses in the excess rotational entropy of the methanol molecules increases until the molecular crossover point, then converges to zero, thus explaining the minimum in the total entropy profile at this point.

We perform a similar analysis of the water molecules in Figure $4 \mathrm{~b}$. Again we find that losses in the excess librational entropy are the dominant factor, although the losses increase with increasing methanol concentration. Indeed the excess librational entropy profile closely tracks the excess volume, suggesting an interpretation based on the system density. We note however that the water molecules actually gain excess librational entropy beyond $x_{\mathrm{MeOH}}=0.70$, so density arguments may not be sufficient. Losses in the excess diffusional entropy shows a harmonic dependence on methanol concentration, closely related to the diffusion constant profile in these mixtures. ${ }^{15}$ Interestingly, the excess rotational entropy losses of the water molecules do not reverse sign at the molecular crossover point as it does for the methanol molecules. It has been shown that water molecules in the bulk liquid rotate by a concerted "jump" mechanism; ${ }^{66}$ thus, our results may simply be due to a breakdown of this mechanism with increasing methanol concentration.

\section{CONCLUSIONS}

We have used molecular dynamics simulations and free energy calculation using the $2 \mathrm{PT}$ method to quantify the thermodynamics factors in water/methanol mixtures. We show that the anomalous entropy profile in this system results primarily from loss of excess librational entropy that is reduced with increasing methanol concentration. We find no evidence of water immobilization or iceberg formation in these systems and that the methanol molecules lose as much as 3 times the excess entropy per molecule as do the water molecules.

A C++ code implementing the 2PT method and compatible with most common simulation packages (LAMMPS, AMBER, GROMACS, etc.) is available upon request from the authors: tpascal@wag.caltech.edu or wag@wag.caltech.edu.

\section{AUTHOR INFORMATION}

\section{Corresponding Author}

*E-mail: tpascal@wag.caltech.edu (T.A.P.); wag@wag.caltech. edu (W.A.G.). Telephone: 626-395-2731. Fax: 626-585-0918.

\section{Notes}

The authors declare no competing financial interest.

\section{ACKNOWLEDGMENTS}

Partial support for this work was from the U.S. National Science Foundation [Grants CTS-0608889 and CTS-0548774]. The MSC computational facilities were provided by ARODURIP and ONR-DURIP. We thank Dr. Shiang-Tai Lin (NTU) and Dr. Mario Blanco (Caltech) for useful discussion on the $2 \mathrm{PT}$ method. Additional computing resources were provided by a generous allocation from the KISTI supercomputing facility.

\section{ABBREVIATIONS}

MD: molecular dynamics

2PT: Two-phase thermodynamics method

$x_{\mathrm{MeOH}}$ : methanol mole fraction

\section{REFERENCES}

(1) Franks, F. Water: A Comprehensive Treatise; Plenum Press: New York, 1972.

(2) McQuarrie, D. A. Statistical Mechanics; University Science Books: Sausalito, CA, 2000. 
(3) Butler, J. A. V.; Thomson, D. W.; Maclennan, W. H. J. Am. Chem. Soc. 1933, 674-686.

(4) Lama, R. F.; Lu, B. C. Y. J. Chem. Eng. Data 1965, 10, 216-219.

(5) Frank, H. S.; Evans, M. W. J. Chem. Phys. 1945, 13, 507-532.

(6) Rezus, Y. L. A.; Bakker, H. J. Phys. Rev. Lett. 2007, 99, 148301.

(7) Soper, A. K.; Finney, J. L. Phys. Rev. Lett. 1993, 71, 4346-4349.

(8) Scatena, L. F.; Brown, M. G.; Richmond, G. L. Science 2001, 292, 908-912.

(9) Dixit, S.; Crain, J.; Poon, W. C. K.; Finney, J. L.; Soper, A. K. Nature 2002, 416, 829-832.

(10) Guo, J. H.; Luo, Y.; Augustsson, A.; Kashtanov, S.; Rubensson, J. E.; Shuh, D. K.; Agren, H.; Nordgren, J. Phys. Rev. Lett. 2003, 91, 157401 .

(11) Laage, D.; Stirnemann, G.; Hynes, J. T. J. Phys. Chem. B 2009, $113,2428-2435$.

(12) Soper, A. K.; Dougan, L.; Crain, J.; Finney, J. L. J. Phys. Chem. B 2005, 110, 3472-3476.

(13) González-Salgado, D.; Nezbeda, I. Fluid Phase Equilib. 2006, 240, 161-166.

(14) Dai, J.; Li, X.; Zhao, L.; Sun, H. Fluid Phase Equilib. 2010, 289, $156-165$.

(15) Ferrario, M.; Haughney, M.; McDonald, I. R.; Klein, M. L. J. Chem. Phys. 1990, 93, 5156-5166.

(16) Paul, S.; Chandra, A. J. Chem. Theory Comput. 2005, 1, 12211231.

(17) Wensink, E. J. W.; Hoffmann, A. C.; van Maaren, P. J.; van der Spoel, D. J. Chem. Phys. 2003, 119, 7308-7317.

(18) Tanaka, H.; Gubbins, K. E. J. Chem. Phys. 1992, 97, 2626-2634.

(19) Weerasinghe, S.; Smith, P. E. J. Phys. Chem. B 2005, 109, 15080-15086.

(20) Lin, S. T.; Maiti, P. K.; Goddard, W. A. J. Phys. Chem. B 2010, 114, 8191-8198.

(21) Lin, S. T.; Blanco, M.; Goddard, W. A. J. Chem. Phys. 2003, 119, $11792-11805$.

(22) Henchman, R. H. J. Chem. Phys. 2007, 126, 064504.

(23) Wang, L.; Abel, R.; Friesner, R. A.; Berne, B. J. J. Chem. Theory Comput. 2009, 5, 1462-1473.

(24) Huang, S.-N.; Pascal, T. A.; Goddard, W. A.; Maiti, P. K.; Lin, S.-

T. J. Chem. Theory Comput. 2011, 7, 1893-1901.

(25) Pascal, T. A.; Lin, S. T.; Goddard, W. A., III. Phys. Chem. Chem. Phys. 2011, 13, 169-181.

(26) Li, Y. Y.; Lin, S. T.; Goddard, W. A. J. Am. Chem. Soc. 2004, 126, $1872-1885$.

(27) Pascal, T. A.; Goddard, W. A.; Jung, Y. Proc. Natl. Acad. Sci. U.S.A. 2011, 108, 11794-11798.

(28) Kumar, H.; Mukherjee, B.; Lin, S.-T.; Dasgupta, C.; Sood, A. K.; Maiti, P. K. J. Chem. Phys. 2011, 134, 124105-124108.

(29) Spanu, L.; Donadio, D.; Hohl, D.; Schwegler, E.; Galli, G. Proc. Natl. Acad. Sci. U.S.A. 2011, 108, 6843-6846.

(30) Zhang, C.; Spanu, L.; Galli, G. J. Phys. Chem. B 2011, 115, $14190-14195$

(31) Teweldeberhan, A. M.; Bonev, S. A. Phys. Rev. B 2011, 83, 134120.

(32) Allen, M. P.; Tildesley, D. J. Computer Simulation of Liquids; Clarendon Press; Oxford University Press: Oxford [England]; New York, 1987.

(33) Eyring, H.; Ree, T. Proc. Natl. Acad. Sci. U.S.A. 1961, 47, 526537.

(34) Carnahan, N. F.; Starling, K. E. J. Chem. Phys. 1970, 53, 600603.

(35) Lin, S. T.; Jang, S. S.; Cagin, T.; Goddard, W. A. J. Phys. Chem. B 2004, 108, 10041-10052.

(36) Pascal, T. A.; Lin, S.-T.; Goddard, W.; Jung, Y. J. Phys. Chem. Lett. 2012, 3, 294-298.

(37) Pascal, T. A.; He, Y.; Jiang, S.; Goddard, W. A. J. Phys. Chem. Lett. 2011, 2, 1757-1760.

(38) Mansoori, G. A.; Carnahan, N. F.; Starling, K. E.; Leland, J. T. W. J. Chem. Phys. 1971, 54, 1523-1525.

(39) Alder, B. J. J. Chem. Phys. 1964, 40, 2724-2730.
(40) Meroni, A.; Pimpinelli, A.; Reatto, L. J. Chem. Phys. 1987, 87, 3644-3646.

(41) Lai, P.-K.; Hsieh, C.-M.; Lin, S.-T. Phys. Chem. Chem. Phys. 2012, 14, 15206.

(42) Jorgensen, W. L.; Tiradorives, J. J. Am. Chem. Soc. 1988, 110, $1657-1666$.

(43) Kaminski, G. A.; Friesner, R. A.; Tirado-Rives, J.; Jorgensen, W. L. J. Phys. Chem. B 2001, 105, 6474-6487.

(44) Berendsen, H. J. C.; Grigera, J. R.; Straatsma, T. P. J. Phys. Chem. 1987, 91, 6269-6271.

(45) Wu, Y.; Tepper, H. L.; Voth, G. A. J. Chem. Phys. 2006, 124, $024503-024512$.

(46) Jorgensen, W. L.; Chandrasekhar, J.; Madura, J. D.; Impey, R. W.; Klein, M. L. J. Chem. Phys. 1983, 79, 926-935.

(47) Abascal, J.; Vega, C. J. Chem. Phys. 2005, 123, 234505.

(48) Plimpton, S. J. Comput. Phys. 1995, 117, 1-19.

(49) Ryckaert, J. P.; Ciccotti, G.; Berendsen, H. J. C. J. Comput. Phys. 1977, 23, 327-341.

(50) Shinoda, W.; Shiga, M.; Mikami, M. Phys. Rev. B 2004, 69, 134103.

(51) Martyna, G. J.; Tobias, D. J.; Klein, M. L. J. Chem. Phys. 1994, 101, 4177-4189.

(52) Parrinello, M.; Rahman, A. J. Appl. Phys. 1981, 52, 7182-7190.

(53) Tuckerman, M. E.; Alejandre, J.; Lopez-Rendon, R.; Jochim, A. L.; Martyna, G. J. J. Phys. A: Math Gen. 2006, 39, 5629-5651.

(54) Hockney, R. W.; Eastwood, J. W. Computer Simulation Using Particles; Taylor \& Francis: New York, 1989.

(55) Plimpton, S. J.; Pollock, R.; Stevens, M. Particle-Mesh Ewald and rRESPA for Parallel Molecular Dynamics Simulations. Proceedings of the Eighth SIAM Conference on Parallel Processing in Scientific Computing; Society for Industrial and Applied Mathematics: Philadelphia, PA, 1997.

(56) Kirkwood, J. G. J. Chem. Phys. 1935, 3, 300-313.

(57) Zwanzig, R. J. Chem. Phys. 1954, 22, 1420-1426.

(58) Widom, B. J. Chem. Phys. 1963, 39, 2808-2812.

(59) Caleman, C.; van Maaren, P. J.; Hong, M.; Hub, J. S.; Costa, L. T.; van der Spoel, D. J. Chem. Theory Comput. 2011, 8, 61-74.

(60) Zhong, Y.; Warren, G. L.; Patel, S. J. Comput. Chem. 2008, 29, $1142-1152$.

(61) Noskov, S. Y.; Lamoureux, G.; Roux, B. J. Phys. Chem. B 2005, 109, 6705-6713.

(62) Zhong, Y.; Patel, S. J. Phys. Chem. B 2010, 114, 11076-11092.

(63) Li, Z.; Scheraga, H. A. Chem. Phys. Lett. 1989, 154, 516-520.

(64) Dixit, S.; Soper, A. K.; Finney, J. L.; Crain, J. Europhys. Lett. 2002, 59, 377-383.

(65) Dougan, L.; Bates, S. P.; Hargreaves, R.; Fox, J. P.; Crain, J.; Finney, J. L.; Reat, V.; Soper, A. K. J. Chem. Phys. 2004, 121, 64566462.

(66) Laage, D.; Hynes, J. T. Science 2006, 311, 832-835.

(67) NIST Chemistry WebBook: NIST Standard Reference Database Number 69; National Institute of Standards and Technology: Gaithersburg, MD , 2000; http://webbook.nist.gov/chemistry/.

(68) Derlacki, Z. J.; Easteal, A. J.; Edge, A. V. J.; Woolf, L. A.; Roksandic, Z. J. Phys. Chem. 1985, 89, 5318-5322.

(69) Goodwin, R. D. J. Phys. Chem. Ref. Data 1987, 16, 799-892.

(70) Simonson, J. M.; Bradley, D. J.; Busey, R. H. J. Chem. Thermodyn. 1987, 19, 479-492. 